\title{
Role of Diffusion Tensor Imaging of the Median Nerve in Carpal Tunnel Syndrome: A Case Control Comparative Study with Electrophysiological Tests and Clinical Assessment
}

\author{
AHMAD M. WAFAIE, M.D.*; KAREEM M. MOUSSA, M.D.*; HODA M. ABBAAS, M.Sc.*; \\ LAMIA M. AFIFI, M.D.** and AYMAN M. MANSOUR, M.D.*** \\ The Departments of Radiology*, Neurophysiology** and Musculoskeletal Surgery***, Faculty of Medicine, \\ Cairo University, Egypt
}

\begin{abstract}
Background: Carpal Tunnel Syndrome (CTS) is the most common peripheral neuropathy of an upper extremity. The diagnosis of CTS is commonly based on findings from the medical history, physical examination, and provocative tests. To confirm the diagnosis, some clinician accepts Electrophysiological Studies (EPS) as the reference standard. DiffusionTensor Imaging (DTI) is a subtype of diffusion-weighted imaging based on measurement of molecular diffusion along multiple directions in space. Directional anisotropy of water diffusibility can be quantified by imaging indices such as Apparent Diffusion Coefficient (ADC) and Fractional Anisotropy (FA) used to characterize the degree of diffusion anisotropy.
\end{abstract}

Aim of Work: The aim of this study is to study the efficacy of diffusion tensor imaging in the diagnosis of carpal tunnel syndrome.

Material and Methods: Twenty-three wrists of 15 healthy subjects and 47 wrists of 31 CTS patients were evaluated with DTI and EPS. FA and ADC of the median nerve were calculated at four locations: Distal radioulnar joint, pisiform bone, hamate bone and hamate hook, and also determined from the whole median nerve. Electrophysiological test, including nerve conduction velocity was also performed for comparison with clinical assessment as a standard of reference.

Results: There was a significant difference between healthy individuals and patients with carpal tunnel syndrome $(p<0.01$ for both $\mathrm{FA}$ and $\mathrm{ADC}$ ). $\mathrm{FA}_{3}$ valuę less than 0.54 and $\mathrm{ADC}$ value more than $1.19 \times 10^{-3} \mathrm{~mm} / \mathrm{sec}$ might be used in the diagnosis of carpal tunnel syndrome. As regards the DTI results, the sensitivity, specificity, negative predictive value, positive predictive value and accuracy were $97.8 \%, 95.6 \%$, $95.6 \%, 97.8 \%$ and $97.1 \%$ respectively.

Conclusion: DTI scan contribute to carpal tunnel syndrome diagnosis on the basis of FA and ADC measurements.

Correspondence to: Dr. Ahmad M. Wafaie, The Department of Radiology, Faculty of Medicine, Cairo University, Egypt
Key Words: Diffusion tensor imaging - Electrophysiological studies - Carpal tunnel syndrome.

\section{Introduction}

THE most common peripheral neuropathy of the upper limb is Carpal Tunnel Syndrome (CTS). It is caused by Median Nerve (MN) entrapment at the level of the Carpal Tunnel (CT). The etiology of CTS include anatomical variations of the CT or by increased contents such as hypertrophied tendons and ligaments, ganglion cysts, or tenosynovitis $[1,2]$

CTS is more common among women in their third and fourth decades and the dominant hand is frequently affected. The most common symptom is tingling pain, sensory disturbances and cramps in the thumb, index, middle finger and the lateral half of the ring fingers. In advanced cases, thenar atrophy and loss of motor function (thumb abduction and opposition) may be seen $[1,3]$.

CTS is commonly diagnosed based on medical history, physical examination and provocative tests. Some clinician uses Electrophysiological Studies (EPS) to further confirm the diagnosis, but in some cases the results may be equivocal due to discrepancies in the different measured parameters. Imaging has the potential to resolve these inconsistencies [4].

Fat-saturated, T2-weighted Magnetic Resonance Imaging (MRI) can reveal morphological changes in CTS patients, such as enlarged median nerve, nerve flattening, increased signal intensity of the nerve, and flexor retinaculum bowing [5]. However, the sensitivity and specificity of MRI findings are low [6] 
Diffusion-Tensor Imaging (DTI) is a subtype of diffusion-weighted imaging based on measurement of molecular diffusion along multiple directions in space, which may enable characterization of micro architecture of biologic tissues [7] Directional anisotropy of water diffusibility can be quantified by indices such as Apparent Diffusion Coefficient (ADC) and Fractional Anisotropy (FA) [8]

Peripheral nerves show a high degree of anisotropic diffusion due to fibrillar alignment of axons with myelin sheaths and compartmentalization of the fiber bundles [7]. In CTS, the median nerve shows an increase in its perpendicular diffusivity which is demonstrated by decreased FA and increased ADC, indicating that diffusion in the tissue has become more isotropic. One explanation for such an observation is edema, which enlarges the extracellular matrix, creating an isotropic environment that cause an increase in perpendicular diffusivity and a decrease in FA $[\mathbf{9 , 1 0}]$.

Aim of the work:

The aim of this study is to investigate the role of DTI in the diagnosis of CTS and correlate between DTI parameters (FA and ADC measurements) and EPS for detecting their sensitivity and specificity in diagnosis of CTS using clinical assessment as standard of reference.

\section{Patients and Methods}

This prospective study was performed between July 2014 and September 2015. This study included 46 subjects distributed in patients and controls groups. In the patients group, we evaluated 47 wrists in 31 CTS patients while in the control group; we evaluated 23 wrists in 15 healthy subjects. This study was approved by our local institutional review board and ethics committee, and also all participants gave written informed consent.

\section{Inclusion criteria:}

- Patients were clinically evident CTS by an expert orthopedic surgeon, and they were presented with typical clinical history, symptoms, and signs of CTS; e.g. intermittent (nightly) numbness, tingling, or burning sensations in the thumb, index finger, middle finger, and the lateral half of the ring finger; atrophy of the thenar muscle.

- Positive Phalen's, Tinel's and Durkan's test results.

- For the control group, they were completely asymptomatic and had no CTS symptoms as well as negative Phalen's, Tinel's and Durkan's test results. There was no previous history of any neurological, musculoskeletal, neuromuscular, endocrinal, metabolic, autoimmune diseases and did not receive any drugs that could affect the nerve conduction as NSAIDs.

\section{Exclusion criteria:}

- Any contraindications for MRI or a previous surgery on the MN at the wrist.

Patients were subjected to the following:

- Clinical assessment.

- Magnetic resonance neurography.

- EPS.

\section{1- Clinical assessment:}

- All participants were subjected to full clinical history taking including: Name, age, sex, dominant wrist, work, history of present illness (onset of time, duration, etc.) and full medical record including: History of neurological, musculoskeletal, neuromuscular, endocrinal, metabolic, autoimmune diseases and drug intake could affect the nerve conduction as NSAIDs.

- Clinical assessment of CTS by Phalen's, Tinel's and Durkan's tests was done for all subjects in the patients and controls groups. In Phalen's test, the patient is asked to hold their wrists in complete and forced flexion for 30-60 seconds. If the patient develops symptoms such as burning, tingling or numb sensation over the thumb, index, middle and ring fingers the test is considered positive and suggests CTS. Tinel's test is performed by lightly percussing over the $\mathrm{MN}$ and is considered positive when it causes tingling in the thumb, index, middle finger and the lateral half of the ring fingers. Durkan's test is done by pressing the examiner thumb over CT and holds pressure for 30 seconds. An onset of pain or paresthesia in the MN distribution within 30 seconds is a positive result of the test.

\section{2- Magnetic Resonance Neurography (MRN): MR imaging technique:}

- Conventional MRI and DTI were performed by using Gyroscan Interna 1.5T Magnet (Philips Medical Systems, Best, Netherlands). The patients were scanned in the prone position with the examined hand extended over the head (superman position). The dorsum of the examined hand lies parallel to the coronal plane of the magnet. The used circular coil was placed over the wrist joint, and was rapped and fixed by rubber bands.

- Details of the pulse sequences used in our study are shown in (Table 1). 
Table (1): Details of different pulse sequences used.

\begin{tabular}{|c|c|c|c|c|c|c|c|c|c|}
\hline $\begin{array}{l}\text { Pulse } \\
\text { sequence }\end{array}$ & $\begin{array}{l}\text { FOV } \\
(\mathrm{mm})\end{array}$ & $\begin{array}{l}\text { Slice thickness } \\
\qquad(\mathrm{mm})\end{array}$ & $\begin{array}{l}\text { Gap } \\
(\mathrm{mm})\end{array}$ & $\begin{array}{c}\mathrm{TE} \\
(\mathrm{msec})\end{array}$ & $\begin{array}{c}\mathrm{TR} \\
(\mathrm{msec})\end{array}$ & Matrix & $\begin{array}{l}\text { Flip } \\
\text { angle }\end{array}$ & $\begin{array}{l}\text { Total sequence } \\
\text { duration }\end{array}$ & $\begin{array}{c}\text { Number of } \\
\text { signals averaged }\end{array}$ \\
\hline - Axial DTI & 140 & 4 & No & 90 & 4600 & $128 \times 128$ & $90^{\circ}$ & $7 \mathrm{~m} \mathrm{49s}$ & 3 \\
\hline - Axial TIWI & 140 & 3 & 0.5 & 20 & 382 & $128 \times 128$ & $90^{\circ}$ & $2 \mathrm{~m} 55 \mathrm{~s}$ & 2 \\
\hline • Axial T2WI & 80 & 2.5 & 0.5 & 100 & 1000 & $160 \times 120$ & $90^{\circ}$ & $2 \mathrm{~m} \mathrm{24s}$ & 1 \\
\hline $\begin{array}{l}\text { - Axial PD with } \\
\text { fat suppression }\end{array}$ & 80 & 2.5 & 0.5 & 30 & 2300 & $228 \times 197$ & $90^{\circ}$ & $3 \mathrm{~m} \mathrm{27s}$ & 1 \\
\hline
\end{tabular}

\section{Image analysis:}

All data were transferred to the manufacturersupplied workstation with Extended MR Workspace software, Version 2.5.3.0 (Philips Medical Systems, Best, Netherland) to perform fiber tracking.

Circular ROIs were placed in the anatomic location of the MN expected on the basis of information from coronal and axial color-coded maps. ROI placement was done at four levels: (1) Distal Radioulnar Joint (DRUJ), (2) Pisiform (proximal CT), (3) Hamate (middle portion of CT) and (4) Hook of hamate (distal CT). The locations of the coded fibers were confirmed by use of the anatomic location on T1-weighted reference images. The tracts were obtained automatically by the software after accurate replacement of the ROIs. ROIs were selected smaller than the nerve to avoid partial volume effects. Quantitative measurements were taken; FA and ADC values obtained from the whole $\mathrm{MN}$ passing through $\mathrm{CT}$ and four focal anatomical sites, from which the mean focal FA and ADC was calculated.

In this study, the interpretation of the quantitative MRN results were considered positive for CTS diagnosis based on our studied statistical Receiver Operating Characteristic (ROC) curves analysis if (1) Mean focal FA is less than or equal 0.54 , and mean foçal ADC is greater than or equal $1.19 \mathrm{X}$ $10^{-3} \mathrm{~mm} / \mathrm{sec}$; and (2) Whole MN FA is less than or equal 0.51, and its $\mathrm{ADC}$ value is greater than or equal $1.23 \times 10^{-3} \mathrm{~mm}^{2} / \mathrm{sec}$.

\section{3- Electrophysiological study:}

All patients and controls had EPS within maximum two weeks of their MR examinations. The EPS adopted in this study included: Motor Nerve Distal Latency (MNDL), Sensory Nerve Distal Latency (SNDL), Motor Nerve Amplitude (MNA) and Sensory Nerve Amplitude (SNA). This is done by transcutaneous stimulating the nerve to create an action potential through an electrical pulse and having the depolarization wave detected by a re- cording electrode that has been placed proximally or distally. Electromyography (EMG) may be defined as the study of the intrinsic electrical activity of muscle, and it is used for assessment of CTS severity at which needles are inserted into the muscle and the electrical activity is studied at rest and during voluntary movements until the maximum contraction is obtained.

The used electrophysiological classification of CTS severity in this study was defined by the American Association of Neuromuscular \& Electrodiagnostic Medicine (AANEM) in 2006 [11] with minor modifications, and it is described as follows: (1) Negative CTS: Normal findings on all MN EPS parameters (including comparative and segmental studies). (2) Early MN entrapment at CT: Abnormal findings only on comparative tests; affected compared SNDL between median $\&$ ulnar nerves of the 4 th digit or median \& radial nerves of the thumb (combined sensory index $>0.5$, and $>0.1$ in cases more than 65 years), and the other EPS parameters were found normal. (3) Mild CTS: Increased SNDL with/out decreased its amplitude, stimulating distance $13 \mathrm{~cm}$ with normal MNDL and its amplitude. (4) Moderate CTS: Increased MNDL with/out decreased its amplitude with abnormal SNDL and its amplitude. (5) Severe CTS: Any of the aforementioned EPS abnormalities with evidence of axonal loss as defined by either absence of sensory response in the finger-wrist tract, $\mathrm{MN}$ amplitude was $<2 \mathrm{mv}$ or positive EMG findings (fibrillation potential with positive sharp waves and neurogenic motor unit potential of abductor pollicis brevis muscle). (6) Extreme CTS: Complete absence of the thenar motor response.

Measurements performed and cut-off points or normal values used in this study were done in reference to Jablecki et al., [12] as follows:

- The normative MN sensory nerve conduction studies over digit 2 are distal latency less than $3.6 \mathrm{~ms}$, amplitude greater than $15 \mu \mathrm{v}$, and conduction velocity greater than $56 \mathrm{~m} / \mathrm{s}$. 
- The normative motor nerve conduction studies of the MN recorded over abductor pollicis brevis muscle are distal latency less than $4.4 \mathrm{~ms}$, amplitude greater than $4 \mathrm{mv}$, and conduction velocity greater than $48 \mathrm{~m} / \mathrm{s}$.

\section{Results}

Thirty-one CTS patients were included in this study, 26 females $(83.87 \%)$ and 5 males (16.12\%). Fifteen healthy controls were also included, 8 females $(53.33 \%)$ and 7 males $(46.66 \%)$. In the patients group, the age ranged between 19 and 84 years with mean age of 46 years and SD of 13.65. In the controls group, the age ranged between 29 and 71 years with mean age of 44 years and SD of 14.31. This study evaluated 47 wrists in 31 CTS patients and 23 wrists in 15 healthy individuals. Regarding patients group, 16 patients had bilateral and 15 patients had unilateral wrist involvement.

\section{1-Electrophysiological results:}

In the patients group, 45 wrists $(95.74 \%)$ had positive EPS results and 2 wrists $(4.25 \%)$ showed negative EPS results. In the controls group, 21 wrists $(95.65 \%)$ wrists had negative EPS results and one wrist (4.34\%) showed positive EPS results. EPS grading in our both groups are shown in (Table 2) and EPS parameters results are shown in (Table 3).

As regards the EPS results in this study, there were 45 true positive, 22 true negative, 1 false positive and 2 false negative result. The sensitivity, specificity, Negative Predictive Value (NPV), Positive Predictive Value (PPV) and accuracy were $95.7 \%, 95.7 \%$, and $91.7 \%, 97.8 \%$ and $95.7 \%$ respectively.

\section{2- MRI results:}

Most of the total examined 47 wrists in our patient group showed no obvious causes for MN entrapment. One patient $(2.12 \%)$ showed small ganglion cyst inside the $\mathrm{CT}$ and two patients (4.24\%) showed bifid median nerve. Mean focal and whole MN DTI results in both groups are shown in (Table 4). A highly significant difference between the DTI parameters between the patients and control group were found at the four focal anatomical sites. A representative case from our control and patient groups is shown in Figs. $(1,2)$ respectively.

ROC curves were performed for mean focal $\mathrm{CT}$ and whole MN DTI values between both groups to calculate their cut-off values in our study. The statistical analysis of those curves is shown in (Table 5).

As regards the DTI results in this study, there were 46 true positive, 22 true negative, 1 false positive and 1 false negative result. The sensitivity, specificity, NPV, PPV and accuracy were $97.8 \%$, $95.6 \%, 95.6 \%, 97.8 \%$ and $97.1 \%$ respectively.

\section{3- Correlation between DTI results and EPS results:}

Details of DTI versus EPS results in patients group are shown in (Table 6). In the patients group, no statistical significant differences were found between the mean focal and whole MN FA and ADC values when comparing the patients in each EPS grade $(p>0.05)$. High significant negative correlation was found between mean focal FA values and EPS grading scale $(r=-0.689, p<0.01)$. However, while whole MN FA values were also negatively correlated with EPS grading scale, the correlation was found not significant ( $r=-0.240$, $p>0.05$ ). Significant positive correlations were found between both mean focal ADC values $(r=0.591, p<0.01)$ as well as whole MN ADC values $(r=0.293, p<0.05)$ and the EPS grading scale.

Table (2): Distribution of EPS grading scale in our study.

\begin{tabular}{llll}
\hline EPS Grading System & \multicolumn{1}{c}{$\begin{array}{c}\text { Controls } \\
\text { group }\end{array}$} & $\begin{array}{l}\text { Patients } \\
\text { group }\end{array}$ \\
\hline No entrapment of the MN & $22(95.65 \%)$ & 2 & $(4.3 \%)$ \\
Early entrapment of the MN & $0(0 \%)$ & 6 & $(12.8 \%)$ \\
Mild entrapment of the MN & $1(4.34 \%)$ & $13(27.7 \%)$ \\
Moderate entrapment of the MN & $0(0 \%)$ & $19(40.4 \%)$ \\
Severe entrapment of the MN & $0(0 \%)$ & 7 & $(14.9 \%)$ \\
Extreme entrapment of the MN & $0(0 \%)$ & 0 & $(0 \%)$ \\
\hline
\end{tabular}

Table (3): EPS values distribution of the total examined wrists in our study.

\begin{tabular}{llllll}
\hline Study group & Value & $\begin{array}{c}\text { MNDL } \\
(\mathrm{ms})\end{array}$ & $\begin{array}{c}\text { SNDL } \\
(\mathrm{ms})\end{array}$ & $\begin{array}{c}\text { MNA } \\
(\mathrm{mv})\end{array}$ & $\begin{array}{c}\text { SNA } \\
(\mu \mathrm{v})\end{array}$ \\
\hline Controls group & Mean & 3.4 & 2.8 & 8.2 & 40.6 \\
& SD & 0.7 & 0.6 & 3.2 & 15 \\
& Median & 3.4 & 2.7 & 7.8 & 43.00 \\
Patients group & Mean & 5.17 & 3.97 & 5.77 & 16.17 \\
& SD & 1.49 & 1.41 & 4.59 & 12.97 \\
& Median & 5.10 & 3.90 & 5 & 12.80 \\
$p$-values & & $<.01$ & $<.01$ & $<.01$ & $<.01$ \\
\hline
\end{tabular}

MNDL : Motor Nerve Distal Latency.

SNDL : Sensory Nerve Distal Latency.

MNA : Motor Nerve Amplitude.

SNA : Sensory Nerve Amplitude. 
Table (4): Distribution of DTI values in both groups in our study.

\begin{tabular}{|c|c|c|c|c|c|}
\hline \multirow{2}{*}{ Site } & \multicolumn{2}{|c|}{$\begin{array}{l}\text { Control } \\
\text { group }\end{array}$} & \multicolumn{2}{|c|}{$\begin{array}{l}\text { Patient } \\
\text { group }\end{array}$} & \multirow{2}{*}{$\begin{array}{c}p- \\
\text { value }\end{array}$} \\
\hline & FA & $\begin{array}{c}\mathrm{ADC} \\
\left(\mathrm{X} 10^{-3}\right)\end{array}$ & FA & $\begin{array}{c}\mathrm{ADC}_{3} \\
\left(\mathrm{X} 10^{-}\right)\end{array}$ & \\
\hline \multicolumn{6}{|c|}{ Distal radioulnar joint: } \\
\hline Mean & 0.66 & 0.98 & 0.53 & 1.18 & $<.01$ \\
\hline SD & 0.06 & 0.15 & 0.08 & 0.26 & \\
\hline Median & 0.66 & 1 & 0.53 & 1.17 & \\
\hline \multicolumn{6}{|c|}{ Pisiform bone: } \\
\hline Mean & 0.61 & 1.06 & 0.43 & 1.37 & $<.01$ \\
\hline SD & 0.07 & 0.14 & 0.07 & 0.16 & \\
\hline Median & 0.60 & 1.06 & 0.42 & 1.39 & \\
\hline \multicolumn{6}{|c|}{ Hamate bone: } \\
\hline Mean & 0.60 & 1.08 & 0.43 & 1.39 & $<.01$ \\
\hline SD & 0.06 & 0.19 & 0.08 & 0.22 & \\
\hline Median & 0.60 & 1.05 & 0.42 & 1.39 & \\
\hline \multicolumn{6}{|c|}{ Hook of hamate: } \\
\hline Mean & 0.60 & 1.07 & 0.44 & 1.37 & $<.01$ \\
\hline SD & 0.07 & 0.19 & 0.09 & 0.25 & \\
\hline Median & 0.61 & 1.12 & 0.43 & 1.43 & \\
\hline \multicolumn{6}{|l|}{ Mean focal: } \\
\hline Mean & 0.62 & 1.05 & 0.46 & 1.32 & $<.01$ \\
\hline SD & 0.05 & 0.13 & 0.06 & 0.14 & \\
\hline Median & 0.61 & 1.03 & 0.45 & 1.33 & \\
\hline \multicolumn{6}{|l|}{ Whole MN: } \\
\hline Mean & 0.58 & 1.12 & 0.45 & 1.34 & $<.01$ \\
\hline SD & 0.05 & 0.13 & 0.05 & 0.10 & \\
\hline Median & 0.58 & 1.14 & 0.45 & 1.35 & \\
\hline
\end{tabular}

Table (5): Cut-off DTI values at the CT level and whole MN in our study.

\begin{tabular}{|c|c|c|c|c|}
\hline & Positive if $\leq$ & AUC & Sensitivi & pecificity \\
\hline \multicolumn{5}{|l|}{$F A$ : } \\
\hline Whole MN & 0.51 & $96.4 \%$ & $91.5 \%$ & $91.3 \%$ \\
\hline Mean focal & 0.54 & $97.3 \%$ & $89.4 \%$ & $95.7 \%$ \\
\hline \multicolumn{5}{|c|}{$A D C\left(X 10^{-3}\right): \quad$ Positive if $\geq$} \\
\hline Whole MN & 1.23 & $94.4 \%$ & $93.6 \%$ & $91.3 \%$ \\
\hline Mean focal & 1.19 & $93.6 \%$ & $89.4 \%$ & $95.7 \%$ \\
\hline
\end{tabular}

Table (6): Details of DTI results versus EPS grading scale in our patient group.

\begin{tabular}{|c|c|c|c|c|}
\hline \multirow{2}{*}{ EPS grading } & \multicolumn{2}{|c|}{$\begin{array}{l}\text { Mean focal DTI } \\
(\text { mean } \pm \text { SD })\end{array}$} & \multicolumn{2}{|c|}{$\begin{array}{l}\text { Whole median nerve } \\
\text { DTI }(\text { mean } \pm \text { SD) }\end{array}$} \\
\hline & FA & $\begin{array}{l}\mathrm{ADC}_{3} \\
\left(\mathrm{X} 10^{-3}\right)\end{array}$ & FA & $\begin{array}{c}\mathrm{ADC}_{3} \\
\left(\mathrm{X} 10^{-3}\right)\end{array}$ \\
\hline \multirow{8}{*}{$\begin{array}{l}\cdot \text { Early entrapment } \\
(\mathrm{n}=6) \\
\cdot \text { Mild CTS } \\
(\mathrm{n}=13) \\
\cdot \text { Moderate CTS } \\
(\mathrm{n}=19) \\
\cdot \begin{array}{l}\text { Severe CTS } \\
(\mathrm{n}=7)\end{array}\end{array}$} & $0.43 \pm$ & $1.36 \pm$ & $0.45 \pm$ & $1.30 \pm$ \\
\hline & 0.08 & 0.23 & 0.05 & 0.06 \\
\hline & $0.48 \pm$ & $1.34 \pm$ & $0.46 \pm$ & $1.28 \pm$ \\
\hline & 0.08 & 0.23 & 0.03 & 0.28 \\
\hline & $0.46 \pm$ & $1.30 \pm$ & $0.44 \pm$ & $1.34 \pm$ \\
\hline & 0.10 & 0.27 & 0.05 & 0.13 \\
\hline & $0.42 \pm$ & $1.38 \pm$ & $0.42 \pm$ & $1.37 \pm$ \\
\hline & 0.07 & 0.22 & 0.06 & 0.04 \\
\hline
\end{tabular}

Figures:

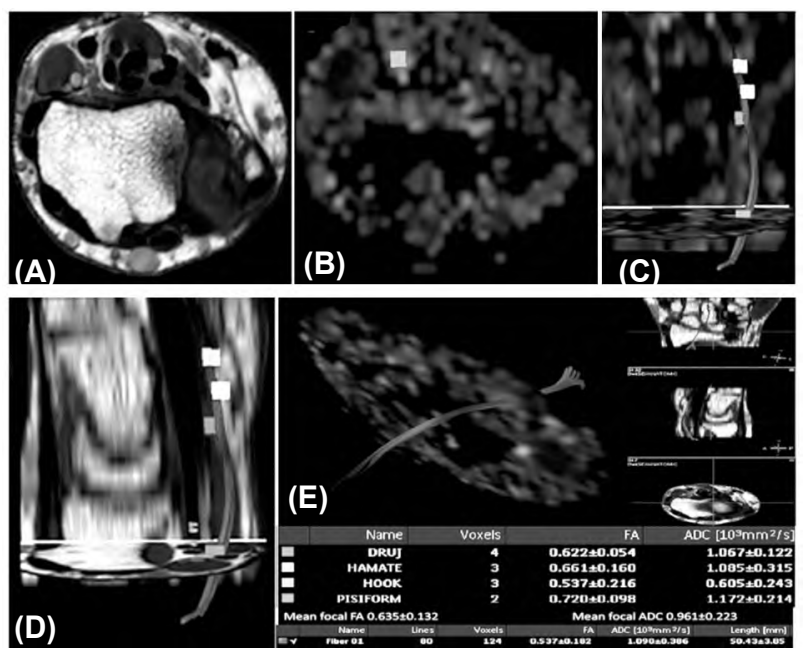

Fig. (1): A 71-year-old male control subject with no history of hand parasthesia or pain as well as no motor weakness. Also he had negative Phalen's, Tinel's and Durkan'stests. EPS investigations are normal. (A) Color coded map image was matched on T1 WIs as an anatomical reference. (B) Color coded map image show a focal ROI was drawn on MN smaller than MN size. (C \& D) sagittal color coded map image and anatomical T1WIs show the whole drawn MN with its focal four anatomical ROIs. (E) DTI results displayed; mean \pm SD values of FA and ADC at the four anatomical sites and their mean values as well the whole MN DTI values were normal.

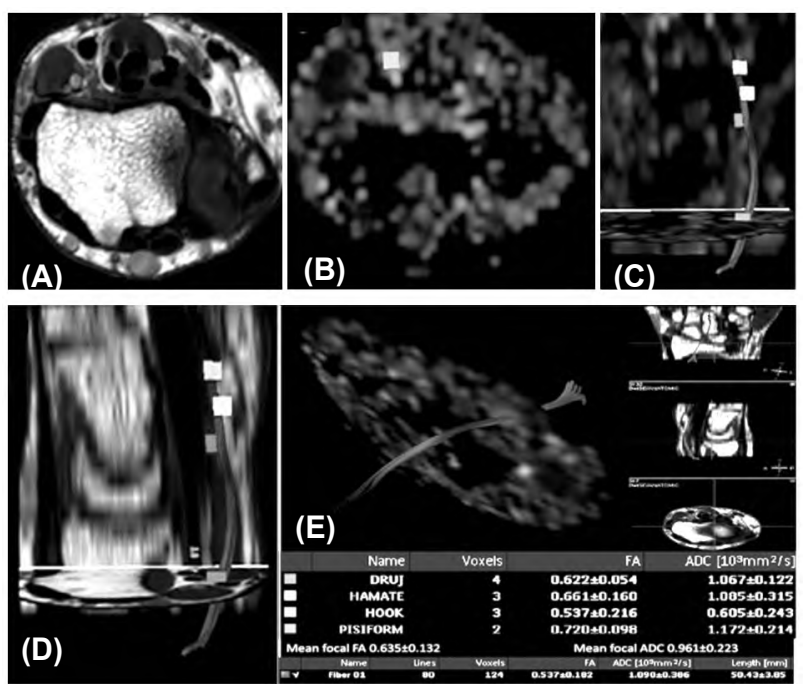

Fig. (2): A 53-year-old female patient with history of hand parasthesia and pain along $\mathrm{MN}$ distribution of her right hand. On clinical examination, she had motor weakness and positive Phalen's, Tinel's and Durkan's tests. EPS investigations showed severe entrapment on MN with positive EMG findings (MNDL $=7.1 \mathrm{~ms}, \mathrm{SNDL}=5.6 \mathrm{~ms}, \mathrm{MNA}=2.1 \mathrm{mv}$ and $\mathrm{SNA}=5.7 \mathrm{v})(\mathrm{A})$ Color coded map image was matched on T1 WIs as an anatomical reference. (B) A color coded map image show a focal ROI was drawn on MN. (C \& D) sagittal color coded map image and anatomical T1WIs show the whole drawn MN with its focal four anatomical ROIs. (E) DTI results displayed entrapment of the $\mathrm{MN}$ at the $\mathrm{CT}$ as mean $\pm \mathrm{SD}$ values of all focal and whole MN FA and ADC were decreased and increased respectively when the MN passed through CT with maximal compression at the level of hamate bone and its hook as both had the lowest FA and highest ADC values respectively. 


\section{Statistical analysis:}

All statistical calculations were done using computer program: Statistical Package for the Social Science (SPSS, Chicago, IL, USA) Version 15 for Microsoft Windows. We described quantitative variables as mean, Standard Deviation (SD) and median and qualitative variables as number and percentage.

\section{The following tests were done:}

- Paired student's $t$-test, ANOVA, Mann-Whitney, Wilcoxon, Post Hoc, Kruskal-Wallis and ChiSquare tests were used to compare quantitative as well as qualitative variables between both groups.

- Spearman's Rho and Pearson correlations were done to evaluate EPS and DTI results with clinical assessment. The sensitivity, specificity, accuracy, positive predictive value, negative predictive value and accuracy were also calculated.

- Probability ( $p$-value):

- $p$-value $<0.05$ was considered significant.

- $p$-value $<0.001$ was considered as highly significant.

- $p$-value $>0.05$ was considered insignificant.

\section{Discussion}

CTS refers to the clinical presentation of sensory abnormalities (pain, numbness and parasthesia) or motor weakness in the $\mathrm{MN}$ distribution [6]. $\mathrm{Al}$ though EPS are considered the single most accurate test that can confirm the diagnosis of CTS with sensitivity of $56 \%$ to $85 \%$ and a specificity of at least $94 \%$, their false negative and false positive rates have been well documented [13,14]. Also EPS have the main drawback of being painful. Therefore, more accurate and painless diagnostic techniques are desirable; MRN by using DTI may provide helpful diagnostic information [15]

In this study, we are trying to assess the DTI as a reliable, noninvasive, recent and accurate method that can investigate the $\mathrm{MN}$ in the $\mathrm{CT}$ in the upper extremity as well as EPS as a most traditional accurate test for CTS diagnosis in relation to the clinical assessment.

The EPS results in this study showed one false positive case in controls group (clinically proved not having nerve entrapment) and two false negative cases in clinically diagnosed CTS patients. The overall accuracy of our EPS results was $95.7 \%$.
A high significant difference was found in comparing the EPS values in-between both groups $(p<0.01)$ in this work. Mean MNDL and SNDL values were found higher in patients group than controls group. While MNA and SNA were found lower in patients group than controls group. MNDL greater than $4.4 \mathrm{~ms}$ is used as a cut-off value for diagnosing CTS in our work. These findings were also noted by Wang et al., [8] who also stated that; the most commonly adopted EPS diagnostic parameter for defining CTS is the MNDL. Larger MNDL indicates more severe CTS, and MNDL greater than $4 \mathrm{~ms}$ was used as a cut-off value for diagnosing CTS.

MR imaging is also helpful in detecting the cause of nerve compression and to identify spaceoccupying lesions. In this study, one patient had obvious intracarpal tunnel ganglion cyst which might be a cause of MN entrapment. Those associated MR findings also studied by Özden et al., [16] who found that two cases out of twelve CTS patients had ganglion cysts as the cause for MN entrapment at the CT.

Two patients with a bifid MN was observed in our study, and this finding was also noted by a study by Granec et al., [17] who found a case of bifid $\mathrm{MN}$ about $3 \mathrm{~cm}$ proximally to the $\mathrm{CT}$. Bifid $\mathrm{MN}$ is not uncommon nerve variation in the CT but it may aggravate CTS symptoms. There are no clinical or electrophysiological tests that could detect anatomical nerve variation in CTS. The knowledge of morphological changes of the MN is important for operative intervention.

According to DTI results in relation to clinical assessment in this work, there was one false positive case in controls group and one false negative case in the patients group. Sensitivity of $97.8 \%$ and specificity of $95.6 \%$ with a total accuracy of $97.14 \%$ were found for MRN in this study.

The distribution of DTI values at the four anatomical regions, mean focal and whole $\mathrm{MN}$ showed a high statistical significant difference between both groups $(p<0.01)$ in this work. The mean focal and whole MN FA values were significantly decreased in CTS patients than controls group while mean focal and whole MN ADC were significantly increased in CTS patients than controls group. The same results were found by the previous publications were a significant difference of mean FA and $\mathrm{ADC}$ values exists between the patients and control groups $[\mathbf{7 , 8 , 1 8 , 1 9 ]}$. In a meta-analysis by Wang et al., [20] FA was significantly lower in CTS patients in comparison with healthy subjects $(p=0.003)$ and 
ADC was significantly higher in CTS patients $(p=0.02)$. Overall sensitivity of FA based diagnosis was $82.82 \%$ and specificity was $77.83 \%$.

On the other hand, Taşdelen et al., [2] reported that CTS patients showed significantly lower FA values in both the whole nerve and focal CT measurements compared to the control group $(p<0.01)$, and no significant difference was observed between ADC measurements.

In this study, mean focal FA and whole MN FA were $0.62 \pm 0.05$ and $0.58 \pm 0.05$ respectively, while $\mathrm{AD}_{-3} \mathrm{C}$ values at the same sites were $1.05+0.13 \mathrm{X}$ $10^{-3} \mathrm{~mm}^{2} / \mathrm{sec}$ and $1.12 \pm 0.13 \times 10^{-3} \mathrm{~mm}^{2} / \mathrm{sec} \mathrm{re}-$ spectively in the control group. On the other hand, CTS patients had a mean focal FA and whole MN FA values of $0.46 \pm 0.06$ and $0.45 \pm 0.05$ respectively while $\mathrm{ADC}$ yalues at the same sites were $1.32 \pm 0.14$ $\mathrm{X} 10^{-3} \mathrm{~mm} / \mathrm{sec}$ and $1.34 \pm 0.10 \times 10^{-3} \mathrm{~mm} / \mathrm{sec}$ respectively. Wang et al., [8] found that mean focal FA and $\mathrm{ADC}_{2}$ values were $0.47 \pm 0.05$ and $1.37 \pm 0.12$ $\mathrm{X} 10^{-3} \mathrm{~mm} / \mathrm{sec}$ respectively in healthy individuals. While in CTS patients, mean FA and ADC values were $0.42 \pm 0.04$ and $1.50 \pm 0.15 \times 10^{-3} \mathrm{~mm}^{2} / \mathrm{sec}$ respectively.

Another study by Taşdelen et al., [2] stated that mean focal FA was $0.59 \pm 0.06$, the mean focal ADC was $1.04 \pm 0.27 \times 10^{-3} \mathrm{~mm}^{2} / \mathrm{sec}$, the whole nerve FA was $0.69 \pm 0.04$ and the whole nerve ADC was $1.03 \pm 0.11 \times 10^{-3} \mathrm{~mm}^{2} / \mathrm{sec}$ in the control group. While in CTS patients; the mean focal FA and $\mathrm{ADC}$ were $0.47 \pm 0.07$ and $1.12 \pm 0.22 \times 10^{-3} \mathrm{~mm} /$ sec respectively, and for the whole nerve; the FA was $0.58 \pm 0.07$ and the ADC was $1.06 \pm 0.13 \mathrm{X}$ $10^{-3} \mathrm{~mm} / \mathrm{sec}$.

The study by Guggenberger et al., [7] also found that in healthy volunteers, FA value ranged from 0.48 to 0.60 and $\mathrm{ADC}$ ranged from 0.956 to 1.095 $\mathrm{X} 10^{-3} \mathrm{~mm} / \mathrm{sec}$ at the different ROIs locations. While in patients with CTS, the FA value ranged from 0.42 to $_{3} 0.53_{2}$ and ADC ranged from 1.069 to $1.201 \times 10^{-3} \mathrm{~mm} / \mathrm{sec}$.

In the study done by Kwon and colleagues [19] FA value ranged from 0.47 to 0,52 and ADC ranged from 1.23 to $1.29 \times 10^{-3} \mathrm{~mm} / \mathrm{sec}$ in the control group. While in patients group, the FA value ranged from 0.4 to 0.43 and $\mathrm{ADC}$ ranged from 1.36 to $1.44 \times 10^{-3} \mathrm{~mm}^{2} / \mathrm{sec}$.

ROC curves were performed for DTI values between both groups to calculate their cut-off values in this work. The threshold for mean focal FA and ADC values were 0.54 and $1.19 \times 10$ $\mathrm{mm}^{2} / \mathrm{sec}$ respectively, and for whole MN FA and
ADC were 0.51 and $1.23 \times 10^{-3} \mathrm{~mm}^{2} / \mathrm{sec}$ respectively.

On the other hand, Guggenberger et al., [7] found that; the threshold for mean focal $\mathrm{FA}_{2}$ and ADC values was 0.47 and $1.05 \times 10^{-3} \mathrm{~mm}^{2} / \mathrm{sec}$ respectively. Our statistical cut-off DTI values were very close to those reached by Taşdelen et al., [2] who reported a cut-off value of 0.55 for mean focal FA and 0.66 for whole-nerve FA at the CT. In a latter study by Kwon et al., [19] the optimal cutoffs were 0.44 for FA and $1.31 \times 10^{-3} \mathrm{~mm} / \mathrm{sec}$ for ADC.

In this work, comparisons were done between DTI results and grading scale of CTS by EPS. Severe CTS patient had a non-significant lower mean focal and whole MN FA values, and nonsignificant higher mean focal and whole MN ADC measurements compared to moderate and mild CTS patients. Similar to a study of Taşdelen et al., [2] the same non-significant findings were also reported except that FA of whole MN value was found significantly lower in severe CTS patients than milder group.

Correlations were done between DTI results and grading scale of CTS by EPS in our work revealing high significant negative correlation between mean focal FA and EPS grading scale and high significant positive correlation between mean focal ADC and EPS grading scale. These observations were also reported by Wang et al., [8] who stated that the linear correlations of FA and ADC versus electrophysiological indicators of CTS were significant. Also, Kwon et al., [19] found that FA and ADC values measured at the carpal tunnel correlated significantly with most of the EPS parameters.

There are few limitations in this study. First, the field strength was only $1.5 \mathrm{~T}$. Use of higher field-strength MR magnets allows for an increased SNR and acquisition of more reliable data from smaller nerves and faster imaging times. Second, FA and ADC threshold values apply only to entrapment neuropathy in patients with CTS. For other neuropathies, as infectious or inflammatory, different threshold values could exist. Third, DTI is technically challenging, and the quality of images depends on field homogeneity, coil as well as the gradient systems used. These technical challenges might hamper the introduction of DTI into the routine clinical practice.

\section{Conclusion:}

DTI enables visualization and characterization of the MN in healthy subjects and CTS patients. 
DTI parameters show clear-cut between the two groups and enable the use of DTI in the diagnosis of CTS.

\section{References}

1- OGE H.K., ACU B., GUCER T., YANIK T., SAVLARLI S. and FIRAT M.M.: Quantitative MRI Analysis of Idiopathic Carpal Tunnel Syndrome, Turk. Neurosurg., 22: 763-8, 2012.

2- TAŞDELEN N., GÜRSES B., KILIÇKESMEZ Ö., FIRAT Z., KARLIKAYA G., TERCAN M., ULUG A.M. and GÜRMEN A.N.: Diffusion tensor imaging in carpal tunnel syndrome, Diagn. Interv. Radiol., 18: 60-6, 2012.

3- KARABULUT Ö., TUNCER M.C., KARABULUT Z. and GÜZE E.: Analysis of MR imaging of wrists in female patients with carpal tunnel syndrome and healthy controls, Int. J. Morphol., 27: 791-800, 2009.

4- GRAHAM B.: The value added by electrodiagnostic testing in the diagnosis of carpal tunnel syndrome, J. Bone Joint Surg. Am., 90: 2587-93, 2008.

5- KOH S.H., KWON B.C., PARK C., HWANG S.Y., LEE J.W. and KIM S.S.: A comparison of the performance of anatomical MRI and DTI in diagnosing carpal tunnel syndrome, Eur. J. Radiol., 83: 2065-73, 2014.

6- ANDREISEK G., CROOK D.W., BURG D., MARINCEK B. and WEISHAUPT D.: Peripheral neuropathies of the median, radial, and ulnar nerves: MR imaging features, Radiographics, 26: 1267-87, 2006.

7- GUGGENBERGER R. MARKOVIC D., EPPENBERGER P., CHHABRA A., SCHILLER A., NANZ D., PRÜSSMANN K. and ANDREISEK G.: Assessment of Median Nerve with MR Neurography by Using Diffusion-Tensor Imaging: Normative and Pathologic Diffusion Values, Radiology, 265: 194-203, 2012.

8- WANG C.K., JOU I.M., HUANG H.W., CHEN P.Y., TSAI H.M., LIU Y.S. and LIN C.C.: Carpal tunnel syndrome assessed with diffusion tensor imaging: Comparison with electrophysiological studies of patients and healthy volunteers, Eur. J. Radiol., 81: 3378-83, 2012.

9- ASSAF Y., FREIDLIN R.Z., ROHDE G.K. and BASSER P.J.: New modeling and experimental framework to characterize hindered and restricted water diffusion in brain white matter, Magn. Reson. Med., 52: 965-78, 2004.

10- SCHWARTZ E.D., COOPER E.T., FAN Y., JAWAD A.F., CHIN C.L., NISSANOV J. and HACKNEY D.B.: MRI diffusion coefficients in spinal cord correlate with axon morphometry, Neuroreport, 16: 73-6, 2005.
11- American Association of Neuromuscular \& Electrodiagnostic Medicine (AANEM), Proper performance and interpretation of electrodiagnostic studies, Muscle Nerve, 33: 436-9, 2006.

12- JABLECKI C.K., ANDARY M.T., FLOETER M.K., MILLER R.G., QUARTLY C.A., VENNIX M.J. and WILSON J.R.: American Association of Electrodiagnostic Medicine; American Academy of Neurology; American Academy of Physical Medicine and Rehabilitation, Electrodiagnostic studies in carpal tunnel syndrome, Report of the American Association of Electrodiagnostic Medicine, American Academy of Neurology, and the American Academy of Physical Medicine and Rehabilitation, Neurology, 58: 1589-92, 2002.

13-LeBLANC K.E. and CESTIA W.: Carpal tunnel syndrome, Am. Fam. Physician; 83: 952-8, 2011.

14- IBRAHIM I., KHAN W.S., GODDARD N. and SMITHAM P.: Carpal tunnel syndrome: A review of the recent literature, Open. Orthop. J., 6: 69-76, 2012.

15- KHALIL C., HANCART C., Le THUC V., CHANTELOT C., CHECHIN D. and COTTEN A.: Diffusion tensor imaging and tractography of the median nerve in carpal tunnel syndrome: Preliminary results, Eur. Radiol., 18: 2283-91, 2008.

16- ÖZDEN R., DUMAN G.A. and YiLD1Z S.O.: SpaceOccupying Lesions: Rare Causes of Carpal Tunnel Syndrome, International Journal of Clinical Research, 2: 127, 2014.

17- GRANEC D., BICANIC G., BORIC' I. and DELIMAR D.: Bifid median nerve in a patient with carpal tunnel syndrome-correlation of clinical, diagnostic and intraoperative findings: Case report and review of the literature, Acta. Clin. Croat., 51: 667-71, 2012.

18- STEIN D., NEUFELD A., PASTERNAK O., GRAIF M., PATISH H., SCHWIMMER E., ZIV E. and ASSAF Y. Diffusion Tensor Imaging of the Median Nerve in Healthy and Carpal Tunnel Syndrome Subjects, J. Magn. Reson. Imaging, 29: 657-62, 2009.

19- KWON B.C., KOH S.H. and HWANG S.Y.: Optimal parameters and location for diffusion-tensor imaging in the diagnosis of carpal tunnel syndrome: A prospective matched case-control study, A.J.R., Am. J. Roentgenol., 204: 1248-54, 2015.

20- WANG H., MA J., ZHAO L., WANG Y. and JIA X.: Utility of MRI Diffusion Tensor Imaging in Carpal Tunnel Syndrome: A Meta-Analysis, Med. Sci. Monit., 22: 736$42,2016$. 


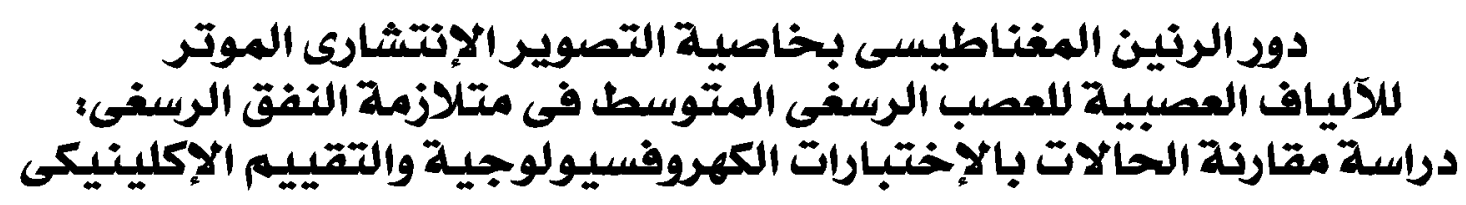

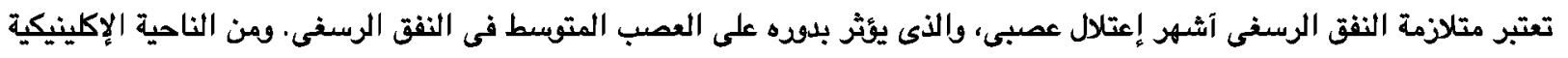

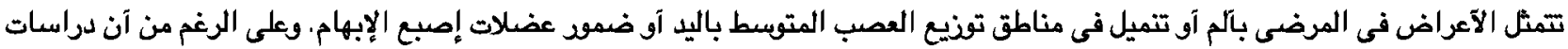

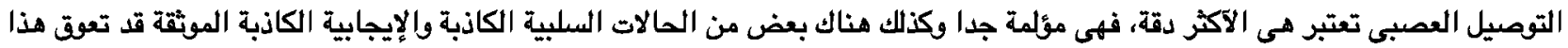

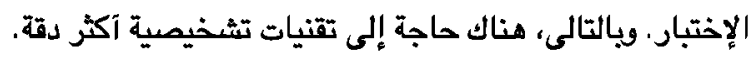

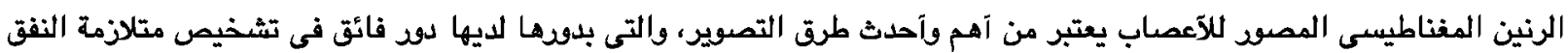

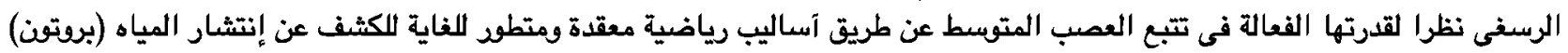

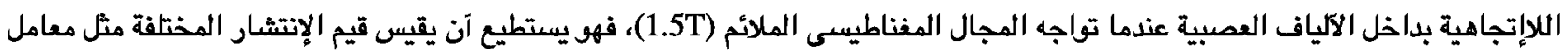

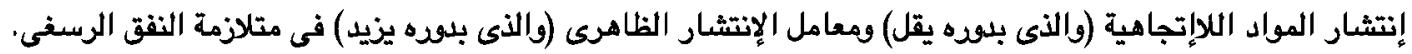

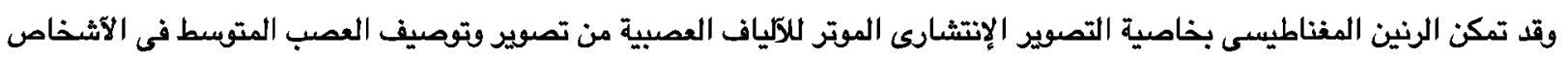

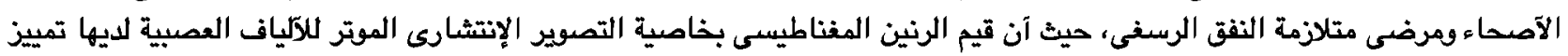

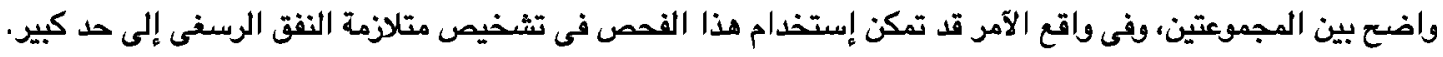

